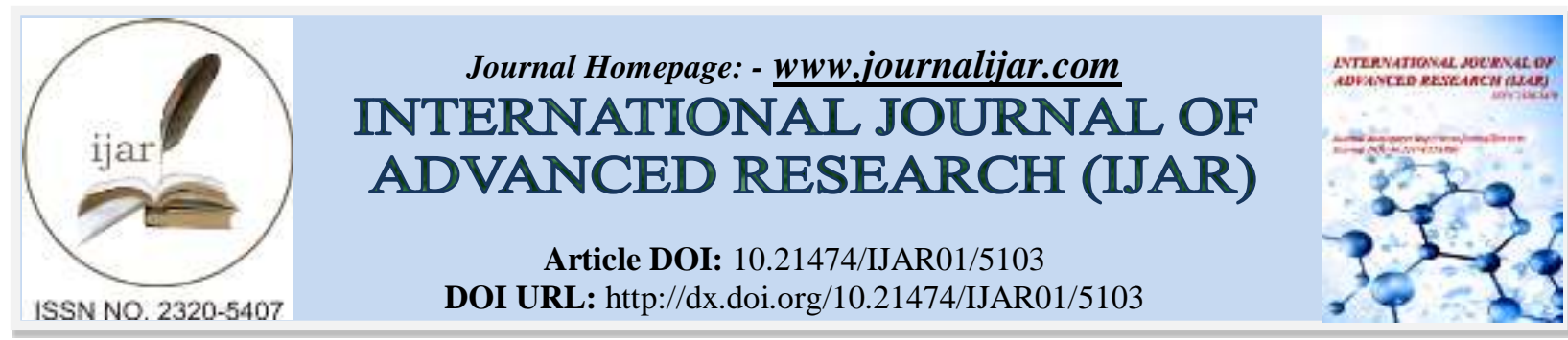

RESEARCH ARTICLE

\title{
A PROSPECTIVE OBSERVATIONAL STUDY COMPARING THE ACCURACY OF APACHE IV AND SAPS II SCORING SYSTEMS IN PREDICTING THE OUTCOME OF ORGANOPHOSPHATE POISONING PATIENTS ADMITTED TO AN INTENSIVE CARE UNIT IN KASHMIR VALLEY.
}

*Dr. Syed Faisal Andrabi, Dr. Sadia Lanker, Dr. Mehraj-ud-din and Dr. Aijaz Ahmed Zargar. Senior Resident, Postgraduate Department of Anaesthesiology, Government Medical College, Srinagar.

\section{Manuscript Info}

Manuscript History

Received: 08 June 2017

Final Accepted: 10 July 2017

Published: August 2017

Key words:-

Ridge splitting, Expansion, Guided bone regeneration.
Abstract

Introduction: Organophosphates are one of the most common causes of poisoning in developing countries, with high morbidity and mortality. As mortality rate of organophosphate poisoning is still high, early diagnosis and appropriate treatment is often lifesaving. Acute poisoning constitutes a significant proportion of Intensive Care Unit (ICU) admissions and even though the overall mortality may be low, they utilise considerable ICU resources. Organophosphates are the main cause of poisoning and death in the Intensive Care Unit of Department of Anaesthesiology and Critical Care, Govt. Medical College Srinagar. Objective: To compare the accuracy of Acute Physiology and Chronic health evaluation IV score (APACHE IV) and Simplified Acute Physiology II score (SAPS II) in the prediction of mortality in patients of organophosphate poisoning admitted to the Intensive Care Unit (ICU) of the Department of Anaesthesiology and Critical Care, Govt. Medical College Srinagar between March 2013 and August 2014. Methods: A prospective study was conducted by collecting data on patients with acute Organophosphate poisoning patients admitted to the Intensive Care unit between March 2013 and August 2014. Data required to calculate the patients' predicted mortality by (APACHE) IV and (SAPS) II scoring systems were collected. Results: A total of hundred (100) patients with organophosphate toxicity who required ICU admission were recruited in the study. The observed mortality following acute organophosphate toxicity in ICU patients was $30 \%$ (30 patients). Predicted mortality by APACHE IV and SAPS II scores were $32 \%$ and $42 \%$ respectively. The area under the receiver operator characteristic (ROC) curves of APACHE IV score $(0.978 \pm$ SE 0.012$)$ was better than of SAPS II score $(0.796 \pm$ SE 0.045) ( $p$ value < 0.05). APACHE IV score of 85 or higher was predictive of mortality with a sensitivity of $93.33 \%$ and specificity of $94.29 \%$, as determined by its ROC curve. And a SAPS II score of 50 or higher was predictive of mortality with a sensitivity of $80 \%$ and a specificity of $74.29 \%$, as determined by its ROC curve. Thus APACHE IV scoring system had a higher sensitivity and specificity in predicting mortality as compared to SAPS II scoring system. Conclusion: APACHE IV and SAPS II scores calculated within the first $24 \mathrm{~h}$ are good prognostic indicators among patients with 
organophosphate toxicity that required ICU admission, with preference to APACHE IV score. APACHE IV and SAPS II scores above 85 and 50 , respectively within the first $24 \mathrm{~h}$ are a predictor of poor outcome in patients with acute organophosphate toxicity.

Copy Right, IJAR, 2017,. All rights reserved.

\section{Introduction:-}

Poison is a substance (solid, liquid or gaseous), which if introduced in the living body, or brought into contact with any part thereof, will produce ill-health or death, by its constitutional or local effects or both. ${ }^{1}$ Acute poisoning is a very common medical emergency both in developed and developing countries of the world. ${ }^{2}$ The exact data of poisoning in India is uncertain due to lack of data at central level as most cases are not reported, and mortality data are a poor indicator of incidence of poisoning. It has been estimated that about 5 to 6 persons per lakh of population die due to poisoning every year in the country. ${ }^{1}$ The commonest cause of poisoning in India and other developing countries is pesticides, the reasons being agriculture based economics, poverty and easy availability of highly toxic pesticides. ${ }^{1}$ Similarly the most common type of poisoning in Kashmir is due to pesticides especially organophosphates, which has been on the rise. ${ }^{2}$

Organophosphates are used as insecticides in agricultural and domestic settings throughout the world. ${ }^{3,5}$ Poisoning with organophosphate compounds is responsible for great morbidity and mortality in developing countries. ${ }^{4,5}$ Intoxication may occur following absorption via gastrointestinal tract, respiratory tract or skin. Absorption through skin may occur in sprayers and handlers. Organophosphates inhibit the enzymes acetyl cholinesterase (AChE) in cholinergic synapses and on red blood cells and butryl cholinesterase in plasma. As a result of this enzyme inhibition, the substrate acetylcholine accumulates. The continued stimulation of the acetylcholine receptor accounts for the clinical signs and symptoms of organophosphate poisoning. ${ }^{3,5}$ The effects/ manifestations produced by these compounds are exaggeration of cholinergic effects - muscarinic, nicotinic and central. ${ }^{2,6}$

Toxicology is integral to critical care practise in India and worldwide. ${ }^{7}$ Scoring systems have been continuously developed to predict outcomes in patients with severe illness, to improve resource allocation and to assist in clinical decision-making particularly for intensive care unit (ICU) patients. ${ }^{5,8}$ According to Gregoire ${ }^{10}$, there are four major purposes of severity-of-illness scoring systems (1) First, scoring systems are used in clinical trials for matching (2) Second, scoring systems are used to quantify severity of illness for administrative decisions such as resource allocation. (3) Third, scoring systems assess ICU performance and compare the quality of care. (4) Fourth, scoring systems are used to assess the prognosis of individual patients. ${ }^{11}$ Such prognostic scoring systems include the Acute Physiology and Chronic Health Evaluation (APACHE) and Simplified Acute Physiology Score (SAPS). ${ }^{5}$

The need to collect quality information on patients in the ICU and to use that information to improve outcomes led to the development of the Acute Physiology, Age, and Chronic Health Evaluation system, known by its acronym APACHE. ${ }^{12}$ Originated at George Washington University by William Knaus, this severity scoring system was first published in 1981. ${ }^{13}$ This initial version of APACHE utilized values from 33 physiologic measurements. Further work by Dr. Knaus and colleagues Dr. Jack Zimmerman, Elizabeth Draper, and Dr. Douglas Wagner led to the introduction of APACHE II in 1985. ${ }^{14}$ APACHE II hospital mortality predictions were based on the prognostic impact of the deviation from normality for 12 physiologic values (Acute Physiology Score or APS), age, chronic health status, and one of 56 disease groups. These variables were used to obtain risk adjusted predictions of mortality that could be utilized for outcome comparisons among Intensive Care Units that were adjusted for differing patient case-mix. In 1991 APACHE III was introduced ${ }^{15}$, changing the number and weightings of APS variables and revising the measurement of chronic health status using seven co-morbidities. Other changes included expanding the number of disease groups to 78 from 56, adding terms for admission source and including a variable indicating if the patient was a post-operative admission. This version of APACHE consisted of a set of equations for predicting ICU and hospital mortality, ICU and hospital length of stay, list of active treatments, duration of mechanical ventilation and the Therapeutic Intervention Scoring System (TISS) score. APACHE IV was published in 2006 to address deterioration in APACHE III performance that had developed despite periodic updating over 15 years. ${ }^{17}$ There were several changes made in this new version of APACHE. The first excluded patients transferred from another Intensive Care Unit from receiving predictions. The second change involved measuring previous length of stay as a continuous rather than an integer variable. And the third change included a variable for designating whether a patient's Glasgow Coma Score could not be assessed due to sedation. The most important 
change involved the new categorization of disease groups. Based on the frequency of selected diagnosis and their mortality rate, the existing 94 disease groups were expanded to $116 .{ }^{16}$

Simplified Acute Physiology Score, developed and validated in France in 1984, used 13 weighted physiological variables and age to predict risk of death in ICU patients. ${ }^{20}$ Like the APACHE scores, SAPS was calculated from the worst values obtained during the first 24 hours of ICU admission. In 1993, Le Gall and colleagues ${ }^{21}$ used logistic regression analysis to develop SAPS II, which includes 17 variables: 12 physiological variables, age, type of admission, and 3 variables related to underlying disease. The SAPS II score was validated using data from consecutive admissions to 137 ICUs in 12 countries. $^{21}$

The aims of this study was to compare the APACHE IV and SAPS II scoring systems in predicting outcome in the Organophosphate intoxicated patients admitted to the Intensive Care Unit of our hospital.

\section{Methods:-}

This clinical study was conducted in the Intensive Care Unit (ICU) of the Postgraduate Department of Anaesthesiology and Critical care of SMHS Hospital, an associated hospital of Government Medical College, Srinagar from March 2013 to August 2014. A prospective observational study was conducted to compare the APACHE IV and SAPS II scoring systems in predicting the outcome in organophosphate intoxicated patients admitted to the Intensive Care Unit.

A total of 100 organophosphate intoxicated patients admitted to the Intensive Care Unit (ICU) over a one and a half year period from March 2013 to August 2014 were included in the study. Patients with age less than 16 years, those who died within four hours of admission to Intensive Care Unit and those who stayed in the Intensive Care Unit for less than $24 \mathrm{hrs}$ were excluded from the study. Data collected included sociodemographic data like age, sex, occupation and residence, time elapsed between acute OP exposure and admission to hospital and ICU and any chronic health condition. A thorough clinical examination and required laboratory investigations of the patients were done. Organophosphate poisoning was confirmed by history, clinical features, toxicological analysis and low serum pseudo-cholinesterase levels. The APACHE IV and SAPS II scores were calculated in accordance with the original methodology, using the worst physiological values in the first ICU day.

The APACHE IV score is made up of the acute physiology score (APS), age and admission circumstances, totaling 142 variables of which 115 are admission diagnoses. The APS is based on the most abnormal values registered during the first $24 \mathrm{~h}$ after ICU admission (such as blood pressure, body temperature, heart rate, etc.).The SAPS II includes only 17 variables: 12 physiology variables, age, type of admission (scheduled surgical, unscheduled surgical, or medical), and three underlying disease variables (acquired immunodeficiency syndrome, metastatic cancer, and hematologic malignancy). ${ }^{5}$

The patients were then observed during their stay in the ICU and the outcome of their condition was noted, whether they survived or died.

Statistical Softwares SPSS (Version 16.0) and MedCalc Statistical were used to carry out the statistical analysis of data. Data was analyzed by means of descriptive statistics viz, percentages, means and standard deviations. Graphically the data was presented by bar and pie diagrams. Student's independent t-test was employed for quantitative data. The Karl Pearson's Chi-square test was used for determination of the relationships between groups (survivors and non-survivors) and categorical variables. A P-value of less than 0.05 was considered statistically significant. The ability and accuracy of models for hospital mortality prediction were determined by examining their discrimination and calibrations. Discrimination power was assessed by the area under the Receiver Operating Characteristic (ROC) curve and calibration by standardized mortality ratio (SMR). SMR was calculated to observe the difference between expected and actual mortality rates as being calculated by dividing observed hospital mortality by the predicted hospital mortality. An Area under curve (AUC) of $>0.9$ was considered to be outstanding, 0.8 to 0.9 excellent, 0.7 to 0.8 acceptable and $<0.7$ was considered poor. The best cut off points, based on the ROC curves, and the sensitivity and specificity of the two scoring systems were calculated. 


\section{Results:-}

A total of 100 patients with acute organophosphate poisoning admitted to ICU were recruited in the study according to inclusion and exclusion criteria. The mortality rate was 30\%. APACHE IV and SAPS II scores were calculated for all 100 patients and compared.

Regarding age of patients, as shown in figure 1 the majority of organophosphate intoxicated patients admitted in Intensive Care Unit were in the age group of 20 to 29 years (45\%) followed by the age group of 16 to 19 years (34\%), $13 \%$ of patients were in the age group of 30 to 39 years, $4.0 \%$ of patients were above 60 years of age, $2 \%$ of patients were in the age group of 40 to 49 years and $2 \%$ of patients in 50 to 59 years age group. The mean age of patients was 24.91( \pm 10.65$)$ years with the youngest patient being 16 years and the eldest patient being 65 years. Relationship between age and survival of organophosphate intoxicated admitted to Intensive Care Unit was statistically insignificant ( $\mathrm{p}$-value $>0.05$ ).

Regarding gender of patient, as shown in figure 2 majority of the organophosphate intoxicated patients admitted to the Intensive Care Unit were Females (68\%) while 32\% were males. Relationship between actual survival and gender of organophosphate intoxicated patients admitted to Intensive Care Unit was statistically insignificant ( $p$ value $>0.05$ ).

Regarding residence of patients, as shown in figure 3 majority of organophosphate intoxicated patients admitted to Intensive Care Unit were from Pulwama (28\%), followed by $21 \%$ from Baramulla, 15\% from Bandipora, 12\% from Srinagar, 7\% from Anantnag, 6\% from Budgam, 5\% from Kupwara, 2\% from Kulgam, 2\% from Ganderbal, and 2\% from Shopian. Majority of organophosphate intoxicated patients admitted to Intensive Care Unit were from rural areas $(64 \%)$ while $36 \%$ of patients were from urban areas (figure 4$)$.

Table -1 depicts the relationship between actual survival and predicted mortality by APACHE IV score and SAPS II score according to their best cut-off point. $32(32 \%)$ patients were predicted to die by APACHE IV score and 26 (26\%) of them were predicted to die by SAPS II score and actually 28 (28\%) patients died, and 68 (68\%) patients were predicted to live by APACHE IV score out of them 16 (16\%) patients only were predicted to die by SAPS II score and actually only $2(2 \%)$ patients died. There was no significant difference between actual survival and nonsurvival patients according to patients SAPS II scores predicted mortality and their APACHE IV scores predicted mortality $(\mathrm{P}>0.05)$.

As depicted in table -2 , the actual mortality in organophosphate intoxicated patients admitted to Intensive Care Unit was 30\% (30/100). Predicted mortality rates were (32\%) and (42\%) for APACHE IV and SAPS II, respectively. Predicted mortality determined by APACHE IV and SAPS II scoring systems was not significantly different from actual mortality. Standardised Mortality Ratio for APACHE IV was 0.94 and for SAPS II was 0.71.

The area under Receiver Operating Characteristic (ROC) curve of the two scoring systems is depicted in figure 5. The area under ROC for APACHE IV was 0.978 (standard error 0.012) and that for SAPS II was 0.796 (standard error 0.045). The difference between the ROC areas of the two scores was 0.182. The area under the curve for APACHE IV was the largest, and there was a statistically significant difference when compared with SAPS II (P < $0.05)$.

The cut-off values, as determined by ROC curves, sensitivities, specificities of the two scoring systems, are depicted in Table 3. An APACHE IV score of 85 or higher was predictive of mortality, as determined by its ROC curve, with a sensitivity of $93.33 \%$ and specificity of $94.29 \%$. A SAPS II score of 50 or higher was predictive of mortality, as determined by its ROC curve, with a sensitivity of $80 \%$ and a specificity of $74.29 \%$.

\section{Discussion:-}

Organophosphate poisoning is a major health problem in developing countries and is often associated with significant morbidity and mortality. ${ }^{22}$ This provision of critical care services consumes a large amount of financial and human resources and has come under scrutiny. ${ }^{23}$ The identification of those who will require prolonged ICU stay or who may be suitable for intermediate (rather than intensive) care may help with the optimal use of limited resources. $^{24}$ 
Large ICU patient datasets and prognostic scoring systems based on them are a valuable part of outcomes research in critical care. ${ }^{9}$ Although prognostic scoring systems have been used to predict the outcome of groups of critically ill patients, the use of a scoring system for prognostication of individual patient outcomes is fraught with difficulty and is controversial. ${ }^{25}$

Two models for predicting outcome in ICU patients have been evaluated in this study. The two models were developed from large heterogeneous cohorts of medical and surgical patients and it is important to evaluate their predictive accuracy in a smaller setting with a different disease spectrum before applying them to make quality of care assessments.

A total of 100 Organophosphate intoxicated patients who were admitted to the ICU were included in the study.

In our study, the majority of organophosphate intoxicated patients admitted to ICU were in the age group of 20 to 29 years $(45 \%)$. Our results were in conformity with study of $\operatorname{Dash}^{28}$ et al (2005) who also observed the highest

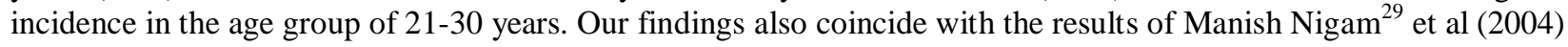
in whose study the commonest age group involved was $21-30$ years.

In our study, majority of the organophosphate poisoning patients admitted to the ICU were Females (68\%). Our findings were similar to Mahrous A. Ibrahim ${ }^{5}$ et al (2011) who also observed majority of organophosphorous poisoning patients admitted to ICU to be females $(55.56 \%)$. Sunder Ram ${ }^{30}$ et al (1991) also observed majority of patients to be females. Our results were not in agreement to the findings of JM Sheikh ${ }^{31}$ (2008) who observed 68.4\% patients to be males and rest females.

In our study, majority of organophosphate intoxicated patients admitted to ICU were from Pulwama district $(28 \%)$.Our results were in conformity to GM Malik ${ }^{32}$ et al who observed that two thirds of the patients of organophosphorous poisoning were from districts with large areas of apple orchards.

In our study, majority of organophosphate poisoning patients admitted to ICU were from rural areas (64\%). Our results were in conformity to Abdul Hakim ${ }^{33}$ et al who observed that most of the acute poisoning patients $(69.23 \%)$ were from the rural background and only $30.77 \%$ of patients were from urban background. However, Mahrous A. Ibrahim $^{5}$ et al (2011) observed that the majority of organophosphorous poisoning cases came from urban areas.

Out of the 100 organophosphate intoxicated patients admitted to the ICU, 70 patients (70\%) survived and 30 (30\%) patients expired. The observed mortality rate in our study was $30 \%$. Our results were in conformity with study of Shadnia $S^{26}$ et al (2007) who observed a mortality of $33.3 \%$ in organophosphorous poisoning patients requiring ICU admission. However, Mahrous A. Ibrahim ${ }^{5}$ et al (2011) observed a mortality of $13.3 \%$ in organophosphorous poisoning patients admitted to their ICU. Syed M Ahmed ${ }^{27}$ (2014) et al observed a mortality of $18.6 \%$.

In our study, the actual mortality in organophosphate intoxicated patients admitted to ICU was 30\%. Predicted mortality rates were $32 \%$ and $42 \%$ for APACHE IV and SAPS II, respectively. Predicted mortality determined by APACHE IV and SAPS II scoring systems was not significantly different from actual mortality. Our study was in agreement with that of Mahrous A. Ibrahim ${ }^{5}$ et al (2011) who stated that the actual mortality in organophosphorous poisoning patients admitted to ICU was $13.3 \%$. They observed that predicted mortality rates were $21.1 \%$ and $23.3 \%$ for APACHE IV and SAPS II, respectively and were not significantly different from actual mortality.

Regarding the standardized mortality ratios, our study showed that the standardized mortality ratios were less than 1 , potentially indicating optimal ICU performance. The APACHE IV and SAPS II SMRs for organophosphorous poisoning patients admitted to the ICU during the period of the study were 0.94 and 0.71 respectively. However, our results were different from the study of Mahrous A. Ibrahim ${ }^{5}$ et al (2011) who observed SMRs of APACHE IV and SAPS II to be 0.67 and 0.53 .

In our study, to estimate the discriminative power of the models, we used the area under the Receiver Operating Characteristic curve. The area under ROC curve for APACHE IV was 0.978 (standard error 0.012) and that for SAPS II was 0.796 (standard error 0.045). The difference between the ROC areas of the two scores was 0.182 . The area under ROC curve for APACHE IV was larger, and there was a statistically significant difference when compared with SAPS II ( $\mathrm{P}<0.05)$. So APACHE IV scoring system is more accurate than SAPS II scoring system in 
predicting outcome in organophosphate intoxicated patients admitted to ICU. Our results are similar to study of Mahrous A. Ibrahim ${ }^{5}$ et al (2011). They observed that the area under ROC curve for APACHE IV was 0.921 (standard error 0.054) and that for SAPS II was 0.807 (standard error 0.078) and that the area under the ROC curve for APACHE IV was greater than that of SAPS II, with the difference being statistically significant.

In our study we observed an APACHE IV score of 85 or higher was predictive of mortality, as determined by its ROC curve, with a sensitivity of $93.33 \%$ and specificity of $94.29 \%$ and a SAPS II score of 50 or higher was predictive of mortality, as determined by its ROC curve, with a sensitivity of $80 \%$ and a specificity of $74.29 \%$. Thus APACHE IV scoring system had a higher sensitivity and specificity in predicting mortality as compared to SAPS II scoring system. Our findings were similar to Mahrous A. Ibrahim ${ }^{5}$ et al (2011) who observed in their study that an APACHE IV score of 89 or higher was predictive of mortality with $93.59 \%$ sensitivity and $91.67 \%$ specificity and a SAPS II score of 44 or higher was predictive of mortality with $85.90 \%$ sensitivity and $75 \%$ specificity. Our results were similar to TA Ayazoglu $^{19}$ (2011) who compared efficacy of APACHE IV and APACHE II scoring systems in predicting outcome of stroke patients admitted to Intensive Care Unit. Area under ROC curve was 0.93 for APACHE IV score. The mean APACHE IV score was 88.7 in their study with a sensitivity of $94.7 \%$ and a specificity of $94.4 \%$ and SMR of 0.95 . APACHE IV predicted mortality was $36.6 \%$ against an observed mortality of $34.54 \%$. Our results were also similar to Mustafa Kamal ${ }^{18}$ et al (2013) who compared APACHE II and APACHE IV scoring systems in predicting outcome in patients with acute lung injury(ALI) and the adult respiratory distress syndrome (ARDS) in intensive care unit (ICU). The area under ROC curve was 0.92 for APACHE IV. The mean APACHE IV score was 90, sensitivity was 94.73\%, specificity was 93.74\% and SMR was 0.94 for APACHE IV score. APACHE IV predicted mortality was $34.04 \%$ against an observed mortality rate of $32 \%$.

Figure 1:-

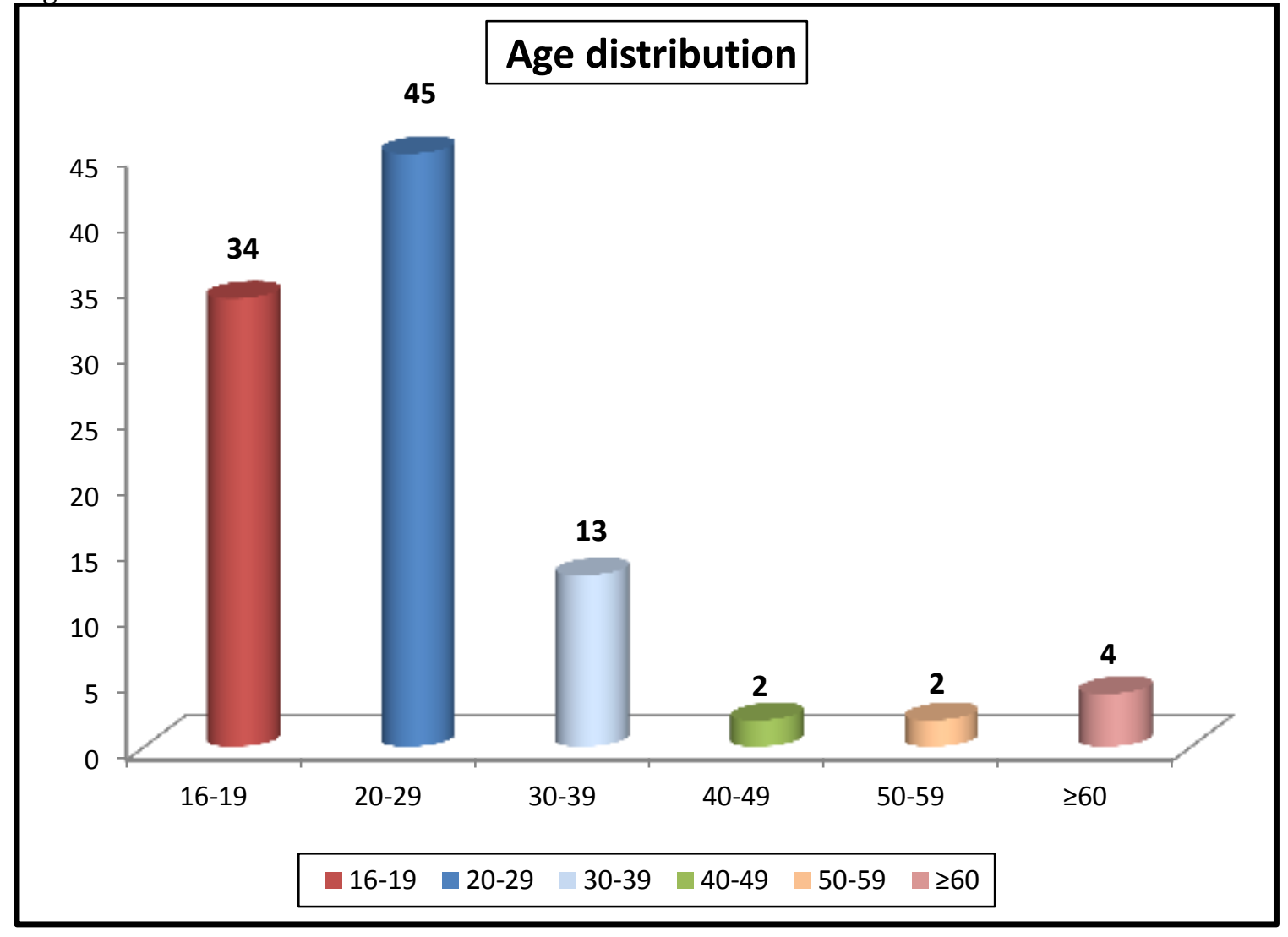


Figure 2:-

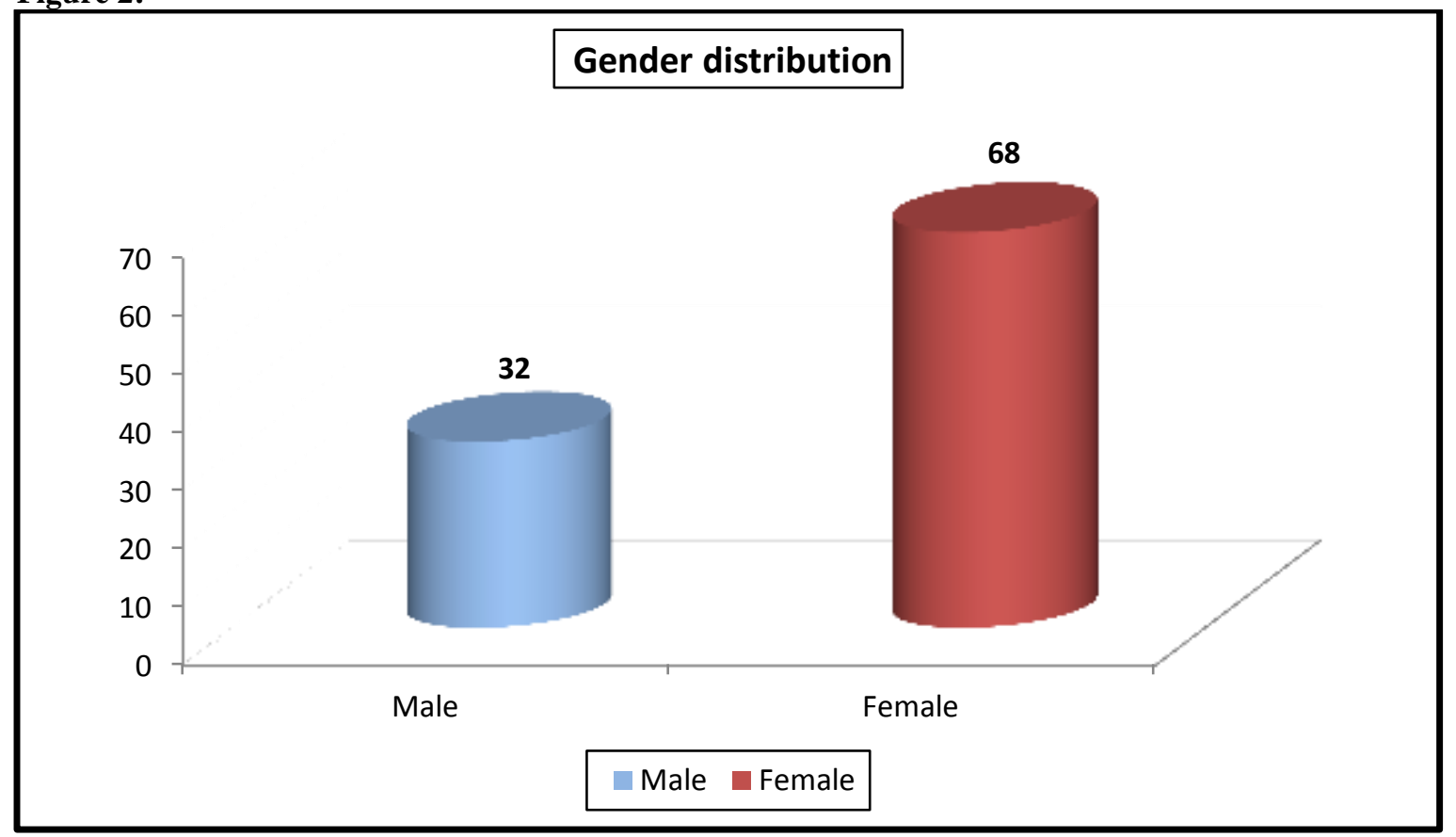

\section{Figure 3:-}

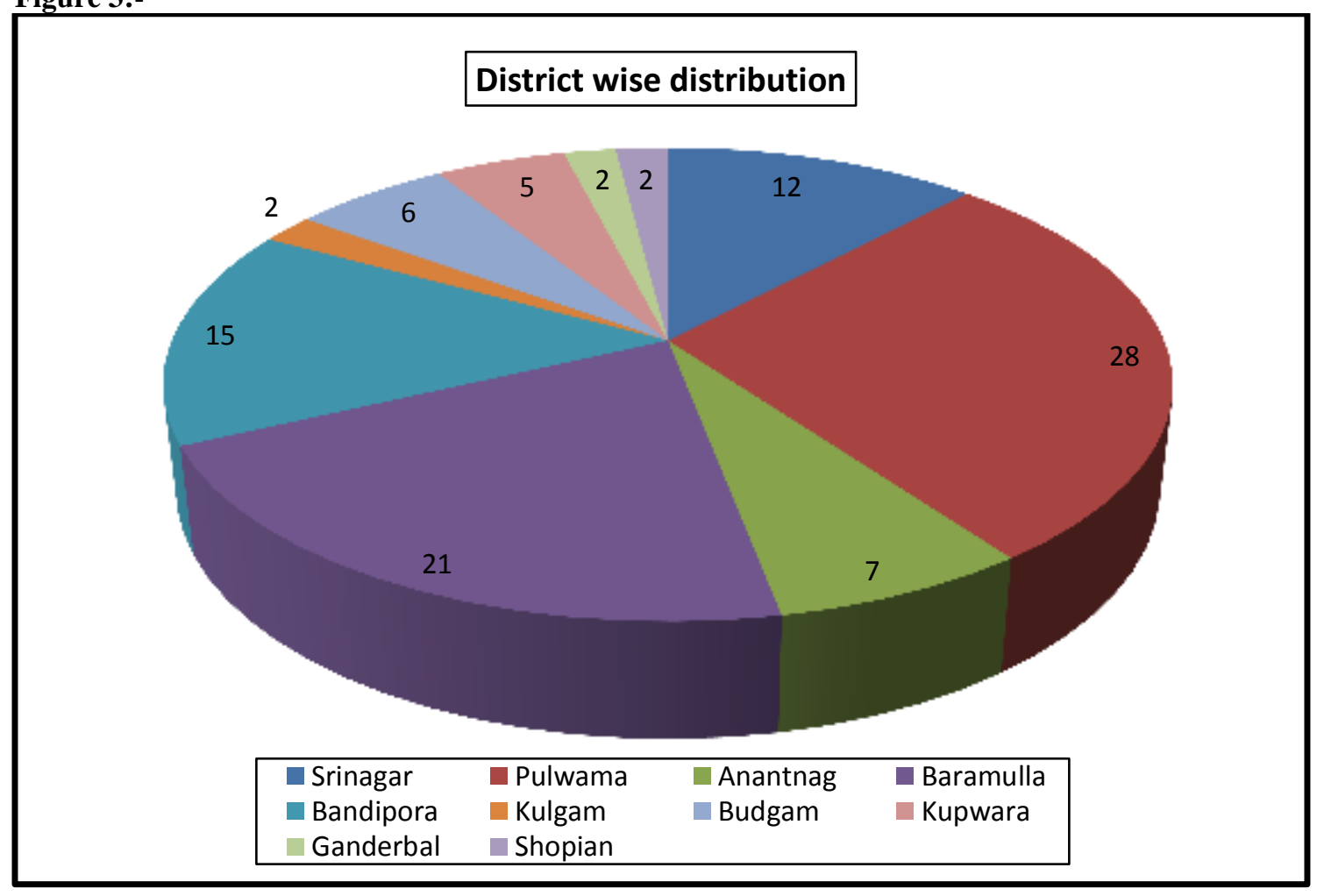


Figure 4:-



Table 1:- Comparison between actual survival and predicted mortality by APACHE IV and SAPS II Score

\begin{tabular}{|c|c|c|c|c|c|c|c|}
\hline \multicolumn{4}{|c|}{ APACHE IV score predicted mortality } & \multicolumn{2}{|c|}{ Actual survival } & \multirow{3}{*}{\begin{tabular}{|l|} 
Total \\
26 \\
\end{tabular}} & \multirow[t]{2}{*}{ P-value } \\
\hline & & & & Non survival & Survival & & \\
\hline \multirow{6}{*}{$\begin{array}{l}\text { Non } \\
\text { survival }\end{array}$} & \multirow{6}{*}{ 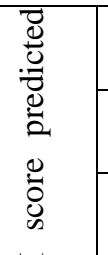 } & \multirow[t]{2}{*}{ Non survival } & No. & 24 & 2 & & \multirow[t]{6}{*}{0.087} \\
\hline & & & \%age & $75 \%$ & $6.3 \%$ & $81.3 \%$ & \\
\hline & & \multirow[t]{2}{*}{ Survival } & No. & 4 & 2 & 6 & \\
\hline & & & \%age & $12.5 \%$ & $6.3 \%$ & $18.8 \%$ & \\
\hline & & \multirow[t]{2}{*}{ Total } & No. & 28 & 4 & 32 & \\
\hline & & & \%age & $87.5 \%$ & $12.5 \%$ & $100 \%$ & \\
\hline \multirow[t]{6}{*}{ Survival } & \multirow{6}{*}{ 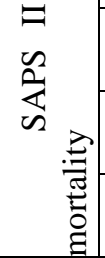 } & \multirow[t]{2}{*}{ Non survival } & No. & 0 & 16 & 16 & \multirow[t]{6}{*}{0.426} \\
\hline & & & \%age & $0 \%$ & $23.5 \%$ & $23.5 \%$ & \\
\hline & & \multirow[t]{2}{*}{ Survival } & No. & 2 & 50 & 52 & \\
\hline & & & \%age & $2.9 \%$ & $73.5 \%$ & $76.5 \%$ & \\
\hline & & \multirow[t]{2}{*}{ Total } & No. & 2 & 66 & 68 & \\
\hline & & & \%age & $2.9 \%$ & $97.1 \%$ & $100 \%$ & \\
\hline
\end{tabular}

Table 2:- Depicting mortalities predicted by the two scoring systems

\begin{tabular}{|l|l|l|l|}
\hline Scoring Systems & Actual mortality (\%) & Predicted mortality (\%) & SMR \\
\hline APACHE IV & 30 & 32 & 0.94 \\
\hline SAPS II & 30 & 42 & 0.71 \\
\hline
\end{tabular}


Figure 5:- Receiver Operating Characteristic (ROC) curve comparison of the APACHE IV and SAPS II scores

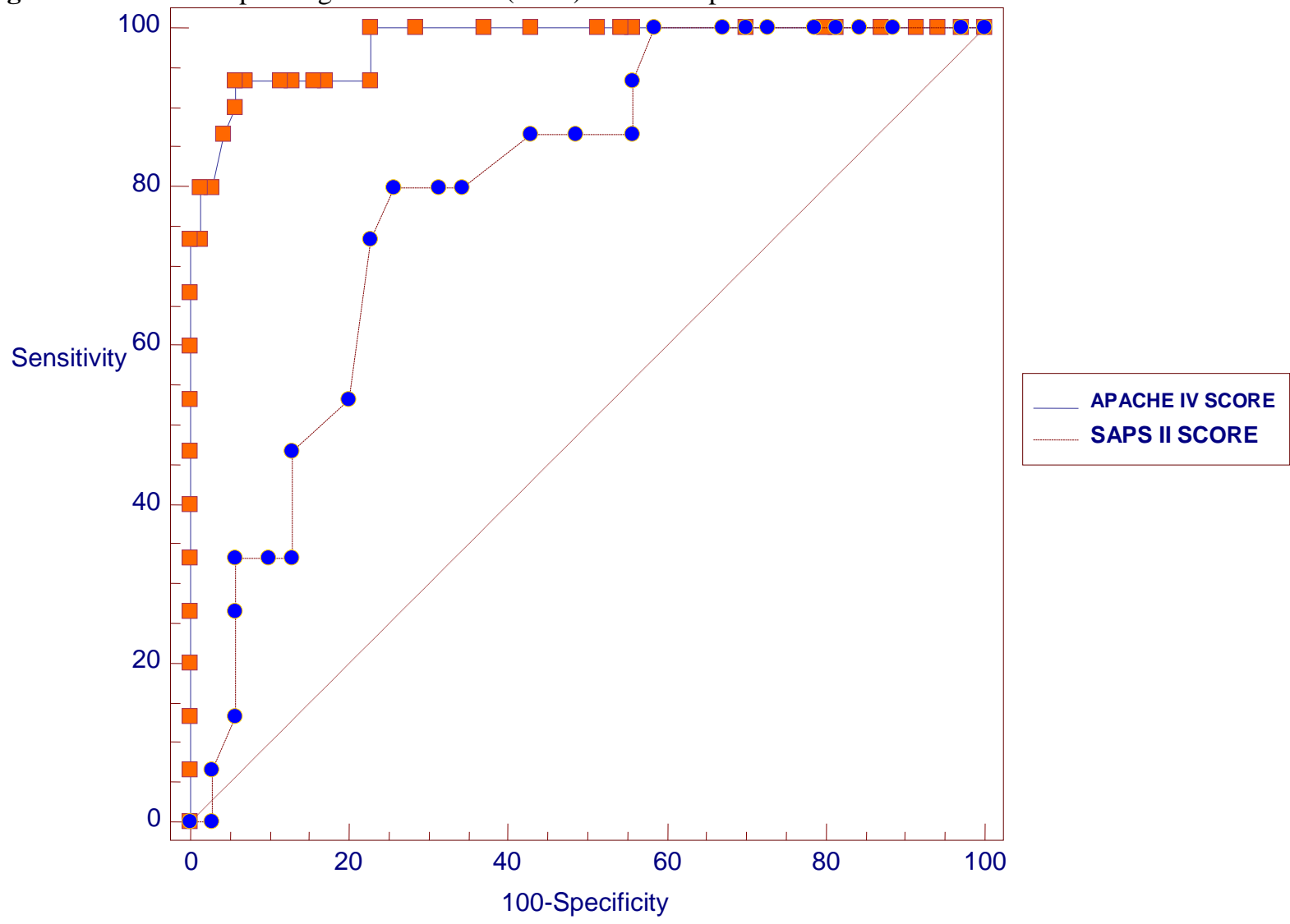

Table 3:- Depicting sensitivity and specificity of two scoring systems

\begin{tabular}{|l|l|l|l|}
\hline Scoring Systems & Best cut-off point & $\begin{array}{l}\text { Sensitivity } \\
(\mathbf{9 5 \%} \text { CI })\end{array}$ & $\begin{array}{l}\text { Specificity } \\
\text { (95\% CI) }\end{array}$ \\
\hline APACHE IV & 85 & 93.33 & 94.29 \\
& & $(77.9-99.2)$ & $(86.0-98.4)$ \\
\hline SAPS II & 50 & 80 & 74.29 \\
& & $(61.4-92.3)$ & $(62.4-84.0)$ \\
\hline
\end{tabular}

\section{Conclusion:-}

Hence based on the data of the present study it is concluded that APACHE IV scoring system is better than SAPS II scoring system in predicting outcome in organophosphate intoxicated patients as observed in our Intensive care unit with a better diagnostic accuracy. Both APACHE IV and SAPS II scoring systems can be used to approximately predict in-hospital mortality, with preference to APACHE IV score.

However, further studies are needed to further develop and validate various other prognosticating scoring systems to help in risk stratification of critically ill patients admitted in the intensive care units and APACHE IV score would be a useful tool for such studies. As the ideal scoring system is yet to be developed and no system has ever been demonstrated to be completely reliable, the ongoing improvement of existing systems should no doubt continue. 


\section{Bibliography:-}

1. Dr. K.S. Narayan Reddy. The Essentials of Forensic Medicine and Toxicology. 2006; $25^{\text {th }}$ Ed: P428, 431.

2. Nikhat Mumtaz; Talib Khan, Shagufta Qazi, Javeed Zargar, Shaqul Qamar. Clinico epidemiological profile of acute organophosphorous poisoning in Kashmir. JK-Practitioner 2008; 15 (1-4): P36-9.

3. Eddleston M, Buckley NA, Eyer P, Dawson AH. Medical management of acute organophosphorus pesticide self-poisoning. Lancet 2008; 371: P597-607.

4. Sivangnanam S. Potential therapeutic agents in the management of organophosphate poisoning. Crit Care 2002;6: P260-1.

5. Mahrous A. Ibrahim, Mohy K. El Masry, Amany A. Moustafa, Abeer M. Hagras, Nahed M. Ali. Comparison of the accuracy of two scoring systems in predicting the outcome of organophosphate intoxicated patients admitted to intensive care unit (ICU). Egyptian J of Forensic Sciences 2011; 1: P41-7.

6. Braunwald. E, Fauci AS. Harrison's Principles of Internal Medicine 15the Ed. Vol-2 : P2614.

7. Henderson A, Wright M, Pond SM. Experience with 732 acute overdose patients admitted to an Intensive Care Unit over six years. Med J Aust 1993; 158(1): P28-30.

8. Rapoport J, Teres D, Lemeshow S, Gehlbach S. A method of assessing the clinical performance and costeffectiveness of intensive care units: a multicenter inception cohort study. Crit Care Med 1994; 22: P1385-91.

9. Higgins T. Severity of illness and outcome prediction: development and evaluation. Textbook of Critical Care 2005: P2195-206.

10. Gregoire G, Russell JA. Assessment of severity of illness. Principle of Critical Care, 1998: P57-69.

11. Chao-Hsiun Tang, Che-Ming Yang, Chi-Yuan Chuang, Ming-Lee Chang, Yu-Chwen Huang, Chin-Feng Huang. A Comparative Study of Clinical Severity Scoring Systems in ICUs in Taiwan. Tzu Chi Med J 2005; 17: P239-245

12. Knaus WA. APACHE 1978-2001: the development of a quality assurance system based on prognosis: milestones and personal reflections. Arch Surg. 137(1): P37-41

13. Knaus WA, Zimmerman JE, Wagner DP, Draper EA, Lawrence DE. APACHE-acute physiology and chronic health evaluation: a physiologically based classification system. Critical Care Medicine. 1981; 9: P591-7.

14. Knaus WA, Draper EA, Wagner DP, and Zimmerman JE. APACHE II: a severity of disease classification system. Critical Care Medicine 1985; 13: P818-29.

15. Knaus WA, Wagner DP, Draper EA, Zimmerman JE, Bergner M, Bastos PG, Sirio CA, Murphy DJ, Lotring T, Damiano A, Harrell FE. The APACHE III prognostic system: risk prediction of hospital mortality for critically ill hospitalized adults. Chest. 1991; 100: P1619-36.

16. L Manganaro, RN and M Stark, MS Cerner Corporation. APACHE Foundations User Guide. Critical Care and Emergency Medicine ABU. September 17, 2010.

17. Thomas 1. Higgins. Textbook of Critical Care $6^{\text {th }} \mathrm{Ed} ; 222$ : P1608.

18. M Kamal, A Naseer Khan, Gauhar Ali. A comparison of APACHE II and APACHE IV scoring systems in predicting outcome in patients with acute lung injury(ALI) and the adult respiratory distress syndrome (ARDS) in intensive care unit (ICU). Rawal Med J 2012; 38(3): P234-8.

19. TA Ayazoglu. A comparison of APACHE II and APACHE IV scoring systems in predicting outcome in patients admitted with stroke to an intensive care unit. Anaesth, Pain \& Intensive Care 2011; 15(1): P 7-12.

20. Le Gall JR, Loirat P, Alperovitch A, Glaser P, Granthil C, Mathieu D, Mercier P, Thomas R. A simplified acute physiology score for ICU patients. Crit Care Med 1984, 12: P975-7

21. Le Gall JR, Lemeshow S, Saulnier F. A new Simplified Acute Physiology Score (SAPS II) based on a European/North American multicenter study. JAMA 1993; 270: P2957-63.

22. Eddleston M. Patterns and problems of deliberate self-poisoning in the developing world. Q J Med 2000; 93(11): P715-31.

23. Angus DC, Shorr AF, White A, Dremsizov TT, Schmitz RJ, Kelley MA. Critical care delivery in the United States: distribution of services and compliance with Leapfrog recommendations. Crit Care Med 2006; 34: P1016-24.

24. Ghosh S, Steyn RS, Marzouk JF, Collins FJ, Rajesh PB. The effectiveness of high dependency unit in the management of high risk thoracic surgical cases. Eur J Cardiothorac Surg 2004; 25(1): P123-6.

25. Le Gall JR. The use of severity scores in the intensive care unit. Intensive Care Med 2005; 31: P1618-23.

26. Shadnia S, Darabi D, Pajoumand A, Salimi A, Abdollahi M. A simplified acute physiology score in the prediction of acute organophosphate poisoning outcome in an intensive care unit. Hum Exp Toxicol. 2007; 26(8): P623-7. 
27. Syed M Ahmed, Bikramjit Das, Abu Nadeem, Rajiv K Samal. Survival pattern in patients with acute organophosphate poisoning on mechanical ventilation: A retrospective intensive care unit-based study in a tertiary care teaching hospital. Indian J Anaesth. 2014; 58(1): P11-7

28. Shreemanta Kumar Dash, AS Raju, Manoj KM, Kiran KM, Sachidananda Mohanty. Sociodemographic profile of poisoning cases. JIAFM 2005; 27(3): P971-3.

29. Manish Nigam, Ashok Kumar, BP Dubey, VK Sharma. Trends of organophosphorous poisoning in Bhopal region: An autopsy based study. JIAFM 2004; 26(2): P971-3.

30. Sunder Ram, SS Kumar and A Jayarajan. Continous infusion of high doses of atropine in the management of organophosphorous compound poisoning. JAPI 1991; 39: P190.

31. JM Shaikh. Management of acute organophosphorous poisoning at a University Hospital. Critical Care. 2008; 12: P357.

32. GM Malik, M Mubarik, GJ Romshoo. Organophosphorus Poisoning in the Kashmir Valley, 1994 to $1997 . \mathrm{N}$ Engl J Med 1998; 338(15): P1078.

33. Abdul Hakim, Rehana Khurshid, Reyaz Ahmed Rangrez Shah, Sameena Mufti, Kewal Krishan, Yudhvir Singh, Dr. Afreen. Pattern, profile and outcome of poisoning cases: A study at a large teaching Hospital in North India. JK Practitioner 2014; 19 (1- 2): P36-40. 